\title{
Application of Interfacial Propagation and Kinking Crack Concept to ECC/Concrete Overlay Repair System
}

\author{
Yaw ChiaHwan and Li Wei \\ Shenyang Architecture and Building University, Hunnan East Road No. 9, Shenyang 110168, China \\ Correspondence should be addressed to Yaw ChiaHwan; 826125581@qq.com
}

Received 1 January 2014; Revised 19 May 2014; Accepted 21 May 2014; Published 23 June 2014

Academic Editor: Juan José del Coz Díaz

Copyright ( 2014 Y. ChiaHwan and L. Wei. This is an open access article distributed under the Creative Commons Attribution License, which permits unrestricted use, distribution, and reproduction in any medium, provided the original work is properly cited.

\begin{abstract}
Research on the application of ultraductile engineered cementitious composite (ECC) as overlay in the repair of deteriorated concrete structures is performed in this paper. Also, interfacial crack kinking and trapping mechanism experimentally observed in ECC/concrete overlay repair system are described by comparison of toughness and energy release rate. The mechanism involves cycles of extension, kinking, and arrest of interfacial crack into the overlay. Experimental testing of overlay repair system reveals significant improvements in load carrying capacity and ductility over conventional concrete overlay. The commonly observed overlay system failure mode of delamination or spalling is eliminated when ECC is applied. These failure modes are suppressed when ECC is used as an ideal and durable candidate overlay repair material.
\end{abstract}

\section{Introduction}

A large number of existing concrete structures worldwide, including previously repaired ones, suffer from deterioration or distress under combined mechanical and environmental loading conditions. These structures are in urgent need of repair. Therefore, it is of research significance and challenge to develop effective and durable repairs, which can fundamentally solve the underlying concrete deterioration problems and protect the old concrete from aggressive environments on the long run.

Concrete repair failure results from a combination of physical, chemical, and mechanical processes. Generally, it is due to repair surface cracking or interface delamination between the overlay and the substrate which are causes of many repair durability problems. Bonded interface between two dissimilar materials is often separated by crack. At certain instance, crack kinks out of the interface and will advance in one of the two materials. It is pointed out in $[1,2]$ that questions, such as whether the crack lies on interface or advances along the interface or would kink out of the interface, are important in the design of the interface between overlay and substrate.
By utilization of analytical solutions and experiments, the present research investigates the performance of ECC as a repair material to improve durability of concrete structures. It is stated [3] that engineered cementitious composite (ECC) is a fiber reinforced cement based composite micromechanically tailored to exhibit metal-like tensile response with ultrahigh tensile strain capacity of 3-5\%, which is about 300 to 500 times the tensile strain capacity of normal concrete and FRC (Figure 1). The superiority of ECC has been brought about by the micromechanics approach and the development in fiber, matrix, interface, and composites processing technology. As demonstrated $[4,5]$, micromechanics relates macroscopic properties to the microstructure of a composite and forms the backbone of material design theory. Especially, it allows systematic microstructure tailoring of ECC as well as material optimization which can lead to extreme composite ductility.

It is reported by Forbes [6] self-healing phenomenon of PVA (polyvinyl alcohol) ECC which is commented as a novel composite to bring revolution to civil engineering. Although failure mode of small scale connections and frames of PVA ECC is analyzed [7], the tremendous cost has limited its application. The cost of PVA ECC is around 5 times that of ECC using PP (polypropylene) fibers. Therefore, it 


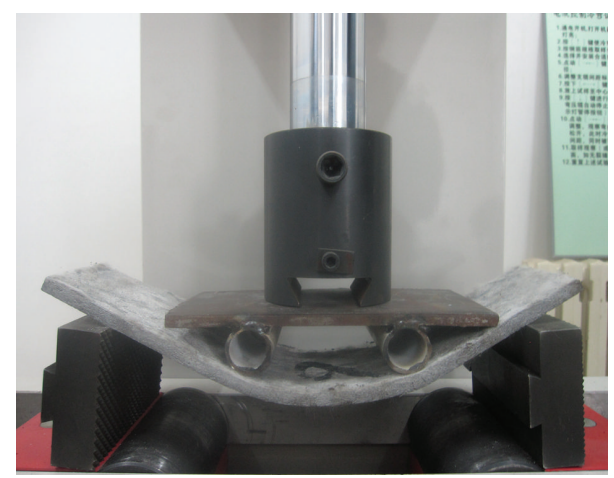

FIgURE 1: Four-point bending test of plain PP ECC.

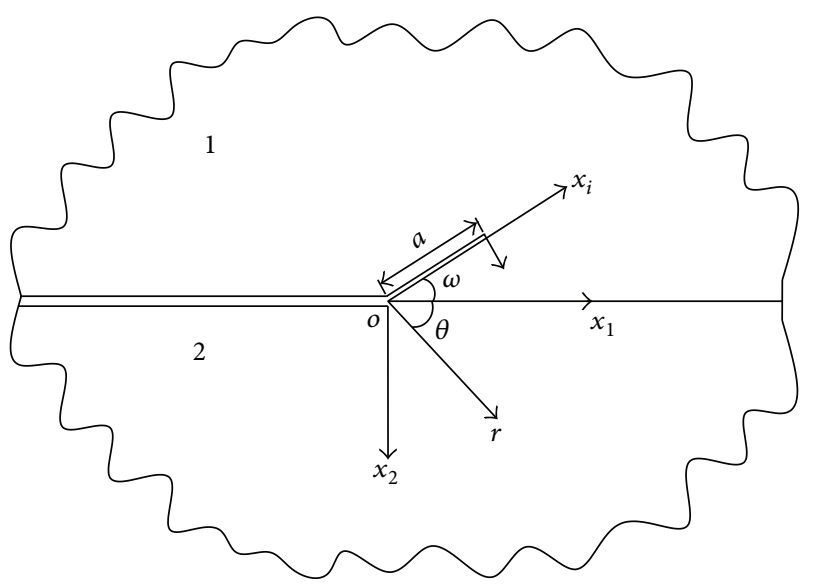

FIGURE 2: Geometry of a crack propagating along and kinking out of the interface.

is necessary to utilize cheap polypropylene fibers to produce ECC with extraordinary properties.

In this paper, findings from experimental and analytical studies carried out on layered repair systems under different surface preparations are reported. For either interpretation of trapping in interface or in the ECC, improvement in mechanical performance is unequivocally demonstrated to exist in the concrete/ECC bimaterial system. The durability of repaired concrete structure using ECC as a repair material is demonstrated in several practical road works.

1.1. Problem Formulation of Propagation and Kinking of Interfacial Crack. For the problem of crack propagating and kinking out of an interface (Figure 2), the two different semiinfinite isotropic elastic materials (overlay as 1 and substrate as 2) are bonded along the positive part of the $x_{1}$-axis. The main crack lies on the interface and a straight crack segment of length $a$ and inclination angle $\omega$ kinks into material 2 .

In overlay repair applications, tensile stress is developed in the repair layer, and a combination of tensile and shear stresses is built up along the interface between the repair and the concrete substrate. Tensile stress at repair/substrate interface is the $x_{2}$ direction stress that opens the interface, which delaminates the interface in the $x_{2}$ direction. Shear stress at repair/substrate interface is the $x_{1}$ direction stress that causes the repair layer to slide along the surface of the substrate, which delaminates the interface in $x$ direction. These stresses can cause repair surface cracking and/or interface delamination. Crack width and delamination magnitude determine the transport properties through this repair system; therefore, they are closely related to repair durability.

The overlay material toughness and interface toughness are $\Gamma_{c}$ and $\Gamma_{i c}$, respectively. Fracture along the interface is more likely to occur than damage of the overlay material if

$$
\frac{\Gamma_{c}}{\Gamma_{i c}}>\frac{G_{0}}{G_{i}}
$$

where $G_{0}$ and $G_{i}$ are energy release rates of kinking crack and interface crack, respectively. The stress intensity factors $K_{I}$ and $K_{I I}$ for the kinked crack and $K_{1}$ and $K_{2}$ for the interfacial crack are dependent on the kinking angle $\omega$ and material moduli. The stress field prior to kinking $(a \rightarrow 0)$ is the singularity field of an interface crack characterized by a complex stress intensity factor:

$$
K=K_{1}+i K_{2} .
$$

1.2. Problem of Interfacial Crack Propagating along the Surface of Bimaterial. The near tip stress field for the specific problem of crack lying along dissimilar isotropic bimaterial interface is a linear combination of two types of fields. The first is coupled oscillatory field defined by $K$ as shown in (2), while the second is nonoscillatory field scaled by a real mode stress intensity factor $K_{3}$ :

$$
\begin{aligned}
\sigma_{i j}=\frac{1}{\sqrt{2 \pi r}} & {\left[\operatorname{Re}\left(K r^{i \varepsilon}\right) \sum_{i j}^{1}(\theta, \varepsilon)+\operatorname{Im}\left(K r^{i \varepsilon}\right) \sum_{i j}^{2}(\theta, \varepsilon)\right.} \\
& \left.+K_{3} \sum_{i j}^{3}(\theta)\right],
\end{aligned}
$$

where $r$ and $\theta$ are polar coordinates and indices $i, j$ refer to coordinates $x_{1}, x_{2}$, and $x_{3}$, respectively.

$\Sigma_{i j}^{1,2,3}(\theta)$ are the angular functions corresponding to tensile stress, in-plane shear stress, and antiplane shear stress across the interface, respectively [8].

The parameter $\varepsilon$ called the bielastic constant or the oscillatory index is given by

$$
\varepsilon=\frac{1}{2 \pi} \ln \left(\frac{1-\beta}{1+\beta}\right) .
$$

The Dundurs parameters are defined, respectively, as

$$
\begin{aligned}
& \alpha=\frac{\mu_{2}\left(\kappa_{1}+1\right)-\mu_{1}\left(\kappa_{2}+1\right)}{\mu_{2}\left(\kappa_{1}+1\right)+\mu_{1}\left(\kappa_{2}+1\right)}, \\
& \beta=\frac{\mu_{2}\left(\kappa_{1}-1\right)-\mu_{1}\left(\kappa_{2}-1\right)}{\mu_{2}\left(\kappa_{1}+1\right)+\mu_{1}\left(\kappa_{2}+1\right)} .
\end{aligned}
$$

Both $\alpha$ and $\beta$ vanish when the materials are identical. $\mu_{i}$ and $\nu_{i}$ are shear modulus and Poisson's ratio, respectively; $\kappa_{i}=3-4 \nu_{i}$ for plane strain and $\kappa_{i}=\left(3-v_{i}\right) /\left(1+v_{i}\right)$ for plane stress. 
TABLE 1: Property of concrete base and repair material.

\begin{tabular}{|c|c|c|c|c|c|c|}
\hline Material & $\begin{array}{c}\text { Elastic modulus } \\
E(\mathrm{Gpa}) \\
\end{array}$ & $\begin{array}{c}\text { Shear modulus } \\
\mu(\mathrm{Gpa})\end{array}$ & Passion's ratio & $\alpha$ & $\beta$ & $\Gamma_{c}[\mathrm{~N} / \mathrm{mm}]$ \\
\hline Concrete base & 25.8 & 10.75 & 0.2 & 0 & 0 & 0.09 \\
\hline $\begin{array}{l}\text { Concrete } \\
\text { overlay }\end{array}$ & 24.9 & 9.96 & 0.25 & -0.006 & 0.019 & 0.09 \\
\hline PVA ECC & 21.3 & 8.24 & 0.27 & -0.012 & 0.013 & 1.587 \\
\hline PP ECC & 18.0 & 6.82 & 0.32 & -0.028 & 0.009 & 1.164 \\
\hline
\end{tabular}

Subscripts 1 and 2 refer to materials 1 and 2, respectively. The complex stresses at distance $r$ ahead of the crack tip take the following form:

$$
\begin{gathered}
\left(\sigma_{22}+i \sigma_{12}\right)_{\theta=0}=\frac{K r^{i \varepsilon}}{\sqrt{2 \pi r}}, \\
\left(\sigma_{23}\right)_{\theta=0}=\frac{K_{3}}{\sqrt{2 \pi r}} .
\end{gathered}
$$

It is clear that $\sum_{i j}^{1,2,3}(\theta)$ correspond to modes 1,2 , and 3 of crack growth. When all three modes are present, the mode mixity is fully specified by two solid angles $\Psi$ and $\phi$ in $\left(K_{1}\right.$, $\left.K_{2}, K_{3}\right)$ space:

$$
\operatorname{tg} \Psi=\frac{K_{2}}{K_{1}}, \quad \cos \phi=\frac{K_{3}}{\sqrt{K_{1}^{2}+K_{2}^{2}+K_{3}^{2}}},
$$

where $\Psi$ is called the phase angle of the complex stress intensity factor or the phase angle of the applied load. It is dependent on applied load, ratio of elastic moduli, and characteristic dimensions of the cracked body.

The complex stress intensity factor for the interfacial crack of the bimaterial is

$$
K=K_{1}+i K_{2} \text {. }
$$

Therefore, it follows that the stress intensity factors are, respectively,

$$
\begin{aligned}
& K_{1}=\operatorname{Re}\left(K h^{i \varepsilon}\right), \\
& K_{2}=\operatorname{Im}\left(K h^{i \varepsilon}\right) .
\end{aligned}
$$

For the special case that $\beta=0$ and $\varepsilon=0, K_{1}$ and $K_{2}$ measure the normal and shear component of the traction singularity acting on the interface.

The stress field on the straight line $\left(x_{1}^{\prime}>0, x_{2}^{\prime}=0\right)$ ahead of the kinked crack tip is characterized by combination of the standard mode $I$ and mode $I I$ stress intensity factors $K_{I}$ and $K_{I I}$ :

$$
\sigma_{2^{\prime} 2^{\prime}}+i \sigma_{1^{\prime} 2^{\prime}}=\left(K_{I}+i K_{I I}\right)\left(2 \pi r^{\prime}\right)^{-1 / 2},
$$

where $x_{1}^{\prime} x_{2}^{\prime}$ is the coordinate system centered at the tip of the kinked crack.

The stress intensity factors of the kinked crack $K_{I}$ and $K_{I I}$ have dimensions of stress (length) ${ }^{1 / 2}$. The relationship between them and the prescribed complex stress intensity factor $K$ can be written as

$$
K_{I}+i K_{I I}=c(\omega) K a^{i \varepsilon}+\bar{d}(\omega) \bar{K} a^{-i \varepsilon},
$$

where $\overline{()}$ denotes complex conjugation. All information for crack kinking out of an interface can be derived from $c(\omega)$ and $d(\omega)$ :

$$
\begin{aligned}
& c(\omega)=\frac{1}{2} \sqrt{\frac{1-\beta}{1+\alpha}}\left(e^{-i \omega / 2}+e^{-3 i \omega / 2}\right), \\
& d(\omega)=\frac{1}{4} \sqrt{\frac{1-\beta}{1-\alpha}}\left(e^{-i \omega / 2}-e^{-3 i \omega / 2}\right) .
\end{aligned}
$$

Since the crack has been taken to kink upward, the loading combinations which result in an opening at both the interfacial and kinking crack tip generally require $K_{I}>0$ and $\Psi \geq 0$.

The relationship between the energy release rate $G_{i}$ of the interfacial crack and complex interface stress intensity factor $K=K_{1}+i K_{2}$ is

$$
G_{i}=\left(\frac{\kappa_{1}+1}{\mu_{1}}+\frac{\kappa_{2}+1}{\mu_{2}}\right) \frac{K \bar{K}}{16 c h^{2}(\varepsilon \pi)} .
$$

The energy release rate $G_{0}$ of the kinked crack $(a>0)$ is given by

$$
G_{0}=\frac{\kappa_{2}+1}{8 \mu_{2}}\left(K_{I}^{2}+K_{I I}^{2}\right)
$$

Using (13), one obtains

$$
G_{0}=\frac{\kappa_{2}+1}{8 \mu_{2}}\left[|c|^{2}+|d|^{2} K \bar{K}+2 \operatorname{Re}\left(c d K^{2} a^{2 i \varepsilon}\right)\right] .
$$

\section{Trapping Mechanism Experiment Verification of the Overlay System}

Besides the PVA and PP ECC/concrete bimaterial system, one additional concrete/concrete system was tested as coupon specimen. All involve base concrete as Material 2, while the overlay systems have concrete, PVA, or PP ECC as Material 1. The concrete (Material 2) was five weeks old when the other material (Material 1) was cast on it. The bimaterial system had two weeks curing before testing. The material compositions and Dundar's elastic mismatch parameters are tabulated in Table 1. The difference of elastic modulus between base 
TABLE 2: The property of four interfaces with different preparation methods.

\begin{tabular}{lcccc}
\hline Specimen number & Repair material & Surface preparation & $\tau_{x y}(\mathrm{Mpa})$ & $\Gamma_{i c}[\mathrm{~N} / \mathrm{mm}]$ \\
\hline 1 & Concrete & Coupon untouched & 0.776 & 0.0772 \\
\hline 2 & PVA ECC & Coupon untouched & 2.287 & 1.002 \\
3 & & Sandblast and moisture & 1.138 & 0.881 \\
4 & PP ECC & Sandblast and scrape & 2.238 & 0.507 \\
\hline
\end{tabular}

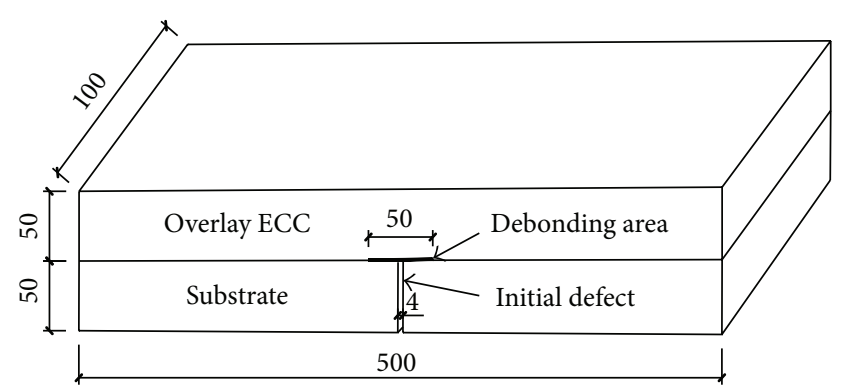

(a)

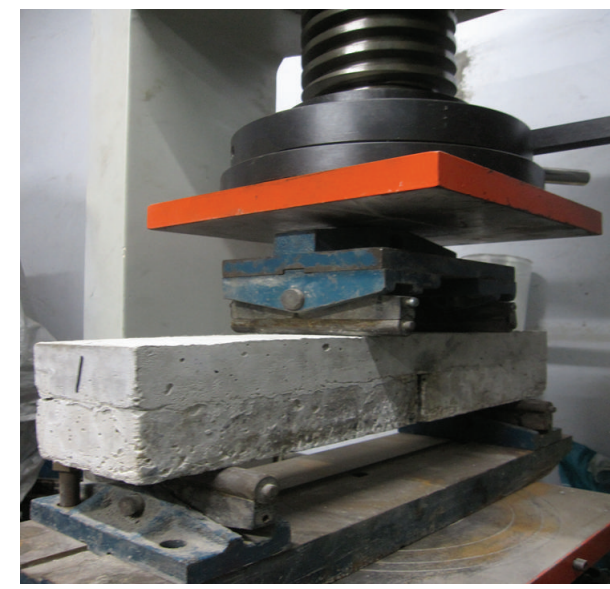

(b)

Figure 3: Test setup.

concrete (Material 1) and concrete for Material 2 is due to differences in their age of curing.

The specimen includes an initial defect of length $50 \mathrm{~mm}$ in the form of an interfacial crack between the base material and the repair Material 1 as well as a joint in the base material. The four-point bending setup is illustrated in Figure 3 and loading speed is $0.3 \mathrm{~mm} / \mathrm{min}$.

Different surface roughening strategies together with a coupon untouched surface were investigated and shown in Table 2. The necessity of having various surface preparation methods is to obtain different bond strengths which are suitable for various requirements of applications.

The different substrate surface roughening techniques exploited during tests are as follows.

(1) Coupon surface is created by the substrate surface as cast against the mould. In fact, it is a mechanically untouched surface for reference purposes.

(2) Sandblast and moisture surface is a roughening of the substrate surface with sandblasting until the coarse aggregate is exposed to about one millimeter clearance. Later, water is put on the surface to make it moist.

(3) Sandblast and scrape surface is a roughening of the substrate surface with sandblasting and then a sharp edged scraping plate is used to scrape the thin, weaker outer layer of the substrate surface and expose the tougher inner material to increase roughness on a micro level. Both the chemical adhesion and

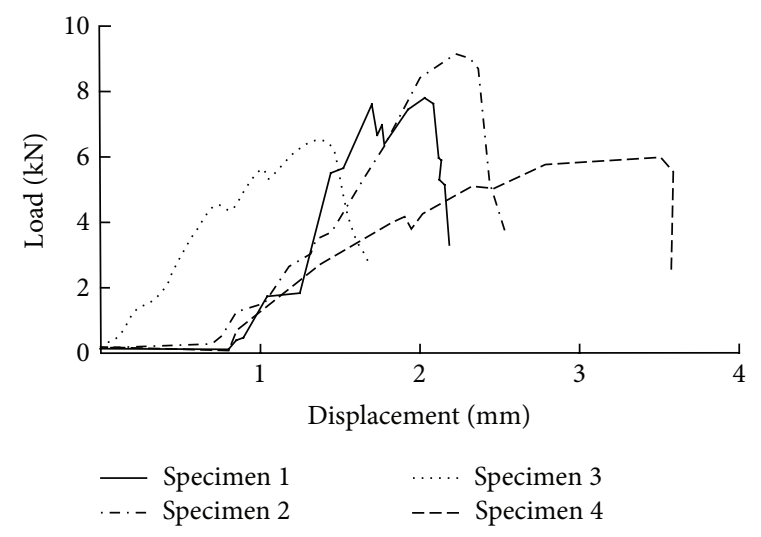

FIGURE 4: Load-deflection curve of four repair overlay specimen: (a) Specimen 1, (b) Specimen 2, (c) Specimen 3, and (d) Specimen 4.

mechanical interlocking between the two materials on a mesoscale are enhanced. The ratio of toughness $\Gamma_{c} / \Gamma_{i c}$ can be measured while the overlay repair system is loaded.

2.1. Experimental Result and Analysis. The load-deflection curve of the four specimens is shown in Figure 4 to compare the effect of different repair material and surface preparation method on the ultimate bearing capacity of the overlay system. It is obvious that the ultimate bearing capacity of Specimen 1 is maximum and reached $9.1325 \mathrm{kN}$. The 


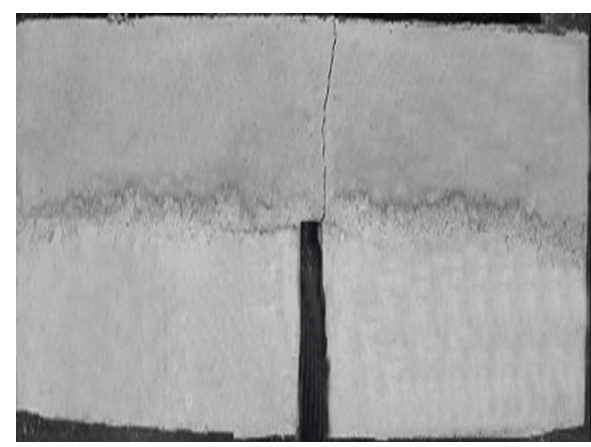

(a)

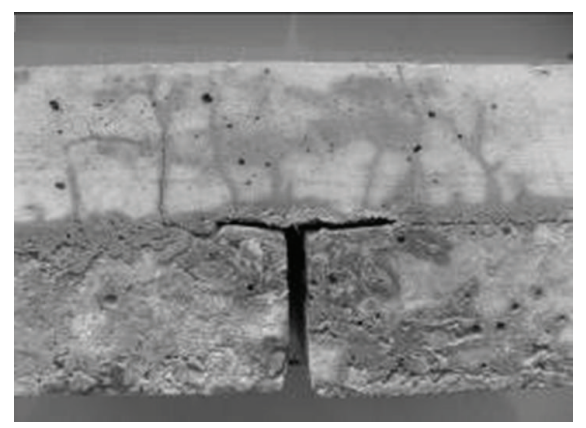

(c)

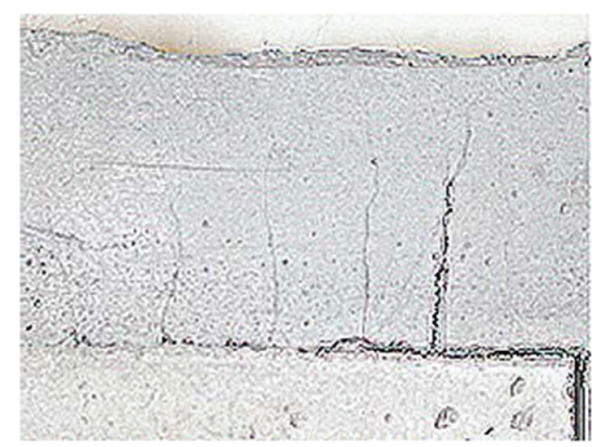

(b)

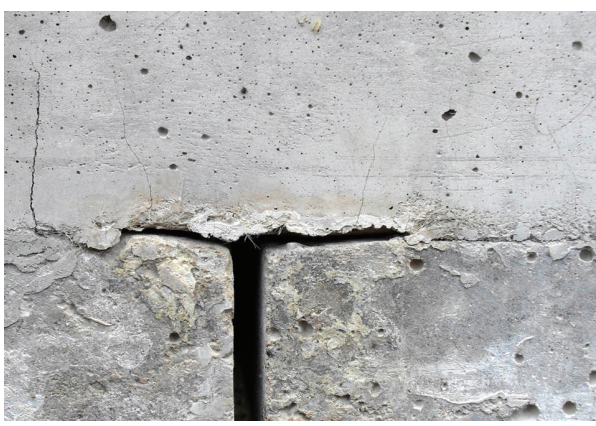

(d)

FIGURE 5: Damage pattern of concrete and ECC repair overlay system. (a) Concrete overlay system, (b) PVA ECC, (c) PP ECC sandblast and moisture, and (d) PP ECC sandblast and scrape.

ultimate bearing capacity of PVA ECC repair overlay system is $7.7935 \mathrm{kN}$. It is shown that the larger the elastic modulus of the repair system, the larger the ultimate bearing capacity. Although the elastic modulus and shear modulus of PP ECC are smaller, the deflection of PP ECC reached $3.3824 \mathrm{~mm}$ and $1.3556 \mathrm{~mm}$, respectively.

As expected, the concrete/ECC bimaterial system shows the distinctive trapping mechanism under this experimental condition. The failure process with trapping mechanism shows tremendous differences compared with those of the control specimens (Figure 5). In Figure 5(a), the concrete/concrete overlay system damaged without obvious deflection and only one macrocrack with large width occurred.

The brittle feature is demonstrated by large crack opening displacement of the kinking crack. The concrete/ECC system shows macroscopic strain-hardening response. The concept of interface crack trapping mechanism is introduced, and its existence is confirmed in experimental investigations. In the concrete/ECC system, the initial interface crack propagated along the interface by about $5 \mathrm{~mm}$, followed by kinking out from the interface. Subsequently, the kinked crack appeared trapped inside the ECC and the mother crack (interface crack) propagated along the interface again (about $27 \mathrm{~mm}$ ). Then, the interface crack kinked and was trapped again. After several times of cracking and kinking, the final failure occurred due to the large opening of a flexural crack in the middle of the specimen. In the PVA ECC overlay system (Figure 5(b)), the angle of kinking crack is around $90^{\circ}$. In the PP ECC overlay system with sandblast and moisture surface (Figure 5(c)), many kinking microcracks emerged around the interface crack and the phase angle is at the range of $45^{\circ}-90^{\circ}$. While the kinking crack number is less for the PP ECC overlay system with sandblast and scrape surface (Figure 5(d)), it is obvious that the overlay system did not absorb much energy during the damaged process. It can be stated that PP ECC with super toughness and ductility can make the overlay system possess more load bearing capacity and durability than common concrete repair overlay. This dramatic improvement in terms of strength, deflection, energy absorption capacity, and ultimate failure mode is not feasible without the trapping mechanism.

2.2. Analysis of Trapping Mechanism. While the phase angle $\Psi$ is small, the value of $\Gamma_{c}$ is also not big. Under this status, the interfacial crack does not kink easily and vice versa. For the experiment performed in this paper, the value of $\Psi$ for the different specimen is 54.2, 28.9, 32.7, and 43.8. In Figure 6, the relationship between $G_{0} / G_{i}$ and kinking angle for the specimen is illustrated.

\section{Conclusions}

By utilization of analytical solutions and experiments, the present research investigates the performance of ECC as a repair material to improve durability of concrete structures. The condition of whether the crack is going to propagate into 


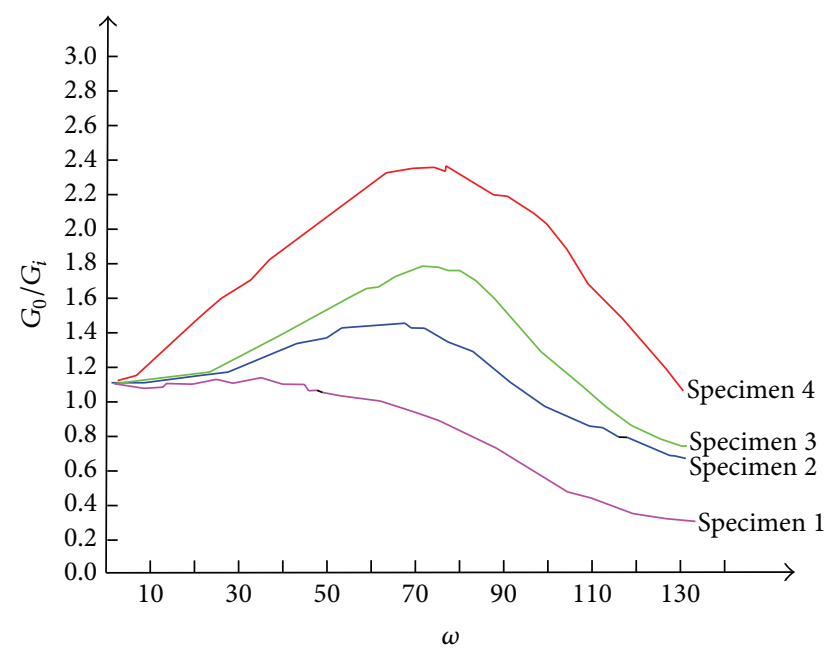

FIGURE 6: Relationship between $G_{0} / G_{i}$ and kinking angle $\omega$.

the substrate or into the upper overlay material is defined. Values of the energy release rate, the stress intensity factor, and mode mixity parameter are determined in terms of dimensionless factor-kinking angle $\omega$, which is a function of geometry and materials elastic properties. The results for the kinked crack can be used to assess whether an interface crack will propagate in the interface or whether it will kink out of the interface. Findings from experimental and analytical studies carried out on layered repair systems under different surface preparations are reported. For interpretation of trapping either in interface or in the ECC, improvement in mechanical performance is unequivocally demonstrated to exist in the concrete/ECC bimaterial system.

\section{Conflict of Interests}

The authors declare that there is no conflict of interests regarding the publication of this paper.

\section{References}

[1] H. Stander, Interfacial Bond Properties for ECC Overlay System [M.S. thesis], University of Stellenbosch, 2007.

[2] T. Nishioka, Z.-D. Zhou, and J.-H. Yu, "Analysis of in-plane transonically propagating interface crack with a finite contact zone," International Journal of Fracture, vol. 142, no. 3-4, pp. 241254, 2006.

[3] J. H. Yu, Properties and Applications of Engineered Cementitious Composites, China Construction Publication House, Beijing, China, 2010.

[4] E. H. Yang, Designing added functions in engineered cementitious composites [Ph.D. thesis], University of Michigan, 2008.

[5] T. Kamada and V. C. Li, "Effects of surface preparation on the fracture behavior of ECC/concrete repair system," Cement and Concrete Composites, vol. 22, no. 6, pp. 423-431, 2000.

[6] A. V. Broek, "Self-healing concrete," Forbes, vol. 184, no. 8, pp. 46-48, 2009.

[7] G. Fischer and V. C. Li, "Effect of matrix ductility on deformation behavior of steel Reinforced ECC flexural members under reversed cyclic loading conditions," ACI Structural Journal, vol. 99, no. 6, pp. 781-790, 2002.

[8] Z. Suo and J. W. Hutchinson, "Interface crack between two elastic layers," International Journal of Fracture, vol. 43, no. 1, pp. 1-18, 1990. 

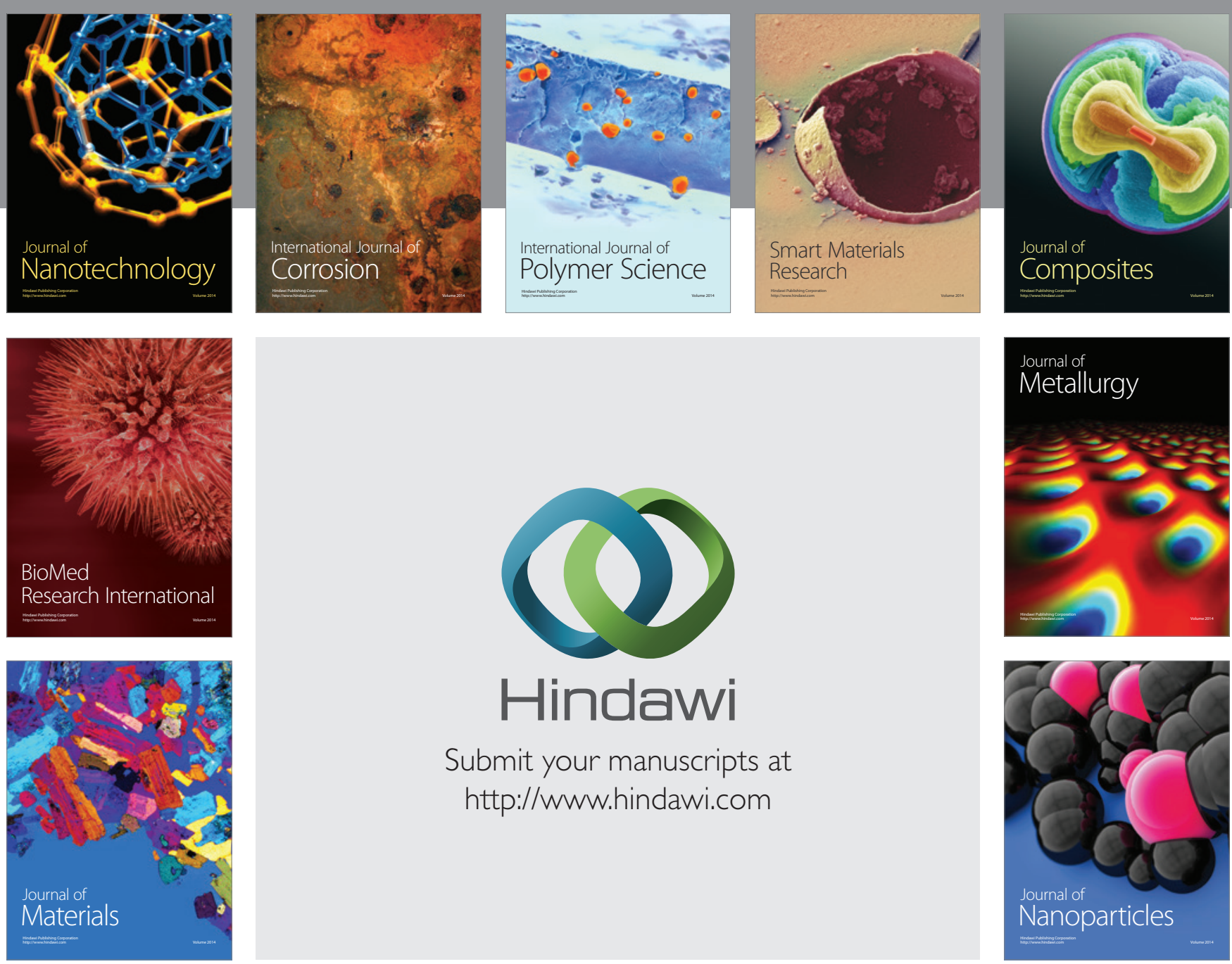

Submit your manuscripts at http://www.hindawi.com
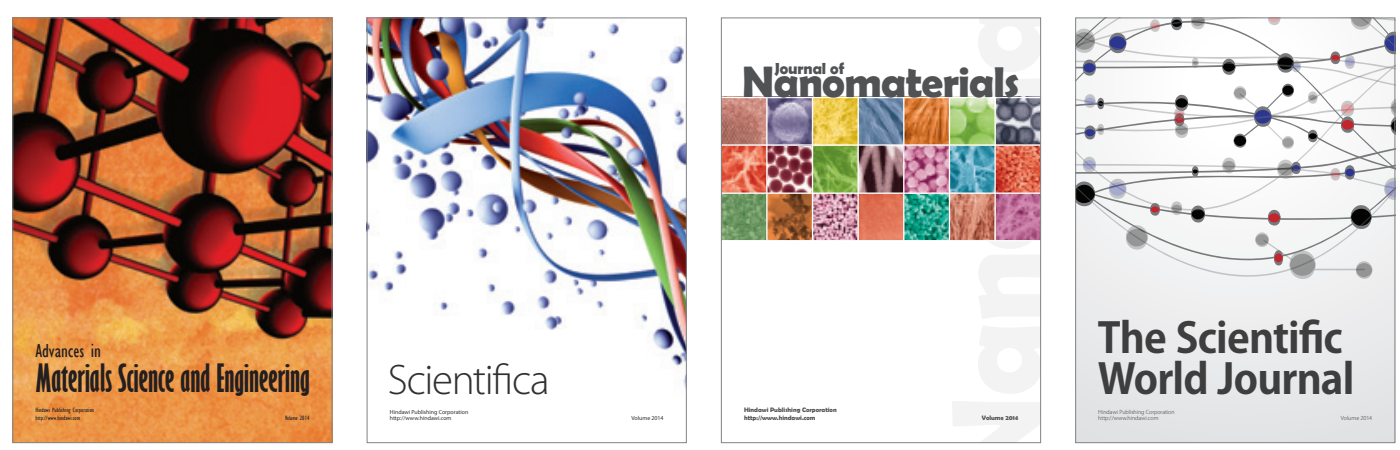

\section{The Scientific World Journal}
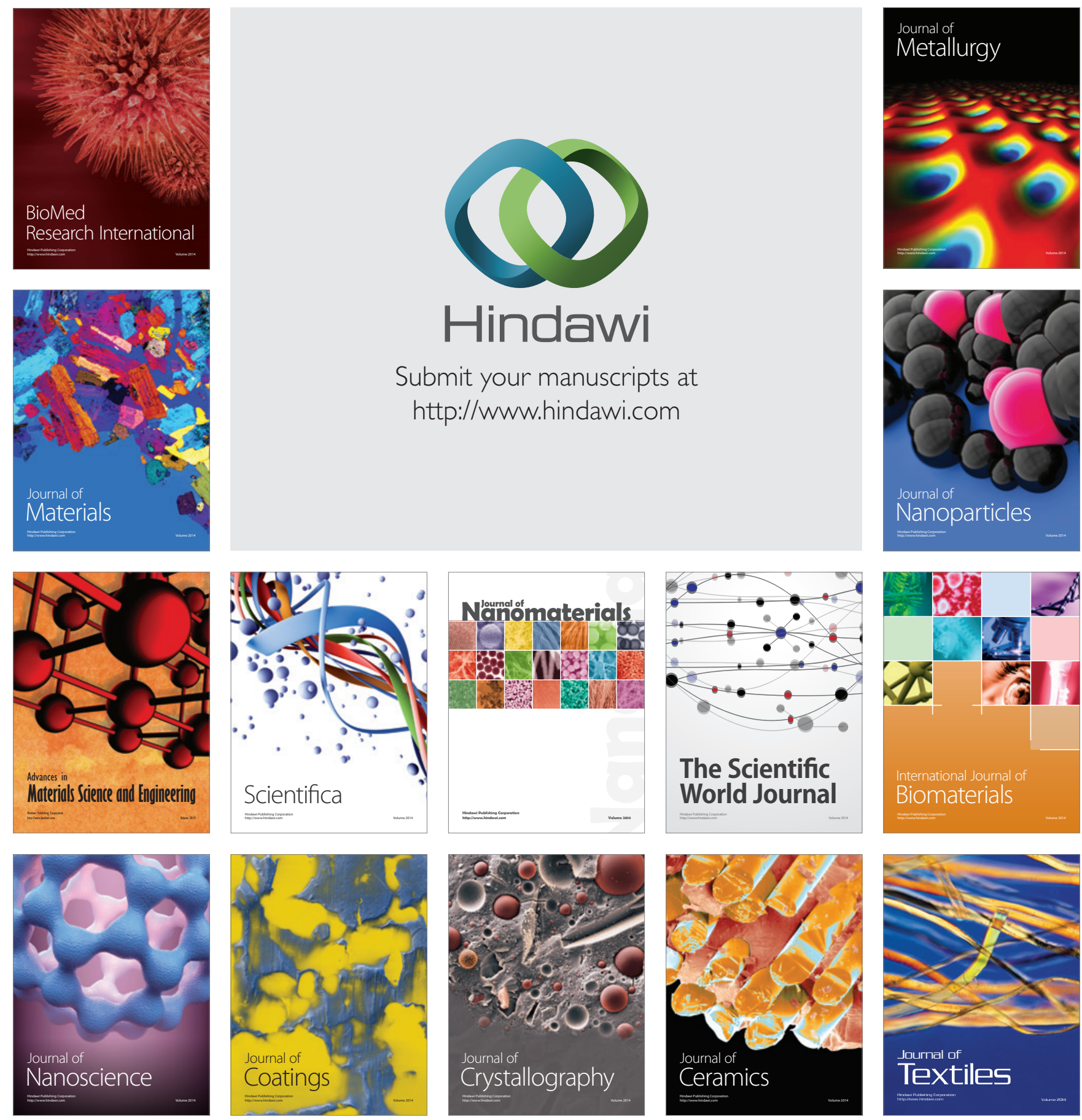